\title{
Die Embryotomie in der Tiergeburtshilfe Versuch zur geschichtlichen Darstellung ihrer Entwicklung
}

\author{
Von Ulrigh Freudiger, Bern
}

Die vorliegende Arbeit soll ein Beitrag zur Geschichte der Tiergeburtshilfe sein. Während die historische Entwicklung der menschenärztlichen Geburtshilfe durch hervorragende Arbeiten abgeklärt ist, stehen wir in der Veterinärmedizin noch ganz am Anfang einer historischen Betrachtung dieses für die tierärztliche Praxis so wichtigen Zweiges, der Tiergeburtshilfe.

Eine genügende Darstellung des Themas gibt es unseres Wissens nicht. Es sind uns nur kurze einleitende Bemerkungen in Lehrbüchern der Tiergeburtshilfe bekannt, die mehr nur eine Aufzählung von Buch- und Autorennamen darstellen.

So war es uns denn unmöglich, für unsere Arbeit über die Embryotomiegeschichte auf schon Bestehendes zurückzugreifen. Wir mußten uns deshalb in die Originalliteratur selbst vertiefen und diese oft mühsam zusammentragen. Deshalb kann die vorliegende Arbeit auch keine das Gebiet erschöpfende, sondern bloß ein Beginn zu weiteren Untersuchungen sein.

Auf Grund der Literaturstudien glauben wir, die historische Entwicklung der Embryotomie in drei große Etappen unterteilen zu können:

1. Etappe: Vom Altertum bis zum Erscheinen des ersten Werkes über Tiergeburtshilfe von Joerg (1818). Diese Etappe mag auf den ersten Blick als zu groß und zu wenig unterteilt scheinen. Sie ist aber dadurch gekennzeichnet, daß die Embryotomie ohne System und nur äußerst selten von besonders geschickten und beherzten Geburtshelfern ausgeführt wurde. Es sind uns nur wenig Literaturhinweise aus dieser Zeit bekannt, die zum Teil den Zeitgenossen unglaubhaft und unmöglich schienen, daß noch 1818 der für die Entwicklung der Tiergeburtshilfe äußerst verdiente Menschenarzt und Leiter der Entbindungsanstalt Leipzig, Dr. Johann Christian GotтFRIED JöRG, ordentlicher öffentlicher Professor der Geburtshilfe an der Universität Leipzig, über eine vom englischen Tierarzt Skellett beschriebene Zerstückelung eines Kalbes im Mutterleibe schrieb: «Es ist dies aber nicht die erste Lüge, die in England ausgesprochen wird.»

II. Etappe : Von 1818 bis ungefähr 1880, oder von Jörg bis zu den Kettensägen. Diese Etappe, mit der wir uns speziell befassen werden, umfaßt im wesentlichen die Entwicklung der subcutanen Methode, des Aus-der-Haut- 
Ziehens, wie die Operation damals genannt wurde. Die Embryotomie wird in dieser Zeit Gegenstand der lehrbuchmäßigen Darstellung. Ein Suchen nach festen Operationsregeln hebt an. Die manuelle Fähigkeit wird durch die Erfindung zahlloser, heute zum größten Teil wieder verschwundener, aber historisch doch bedeutsamer Instrumente unterstützt. Diese Epoche läßt sich unterteilen:

a) Die Zeit der Amputationen, hauptsächlich bis etwa 1830. Es ist dies eine Festigung der schon in der ersten Etappe vereinzelt geübten Abtrennung von Gliedmaßen. Hierzu wurden viele absonderliche Instrumente konstruiert. Sie ist gefährlich und wurde meist nur außerhalb der Gebärmutter geübt.

b) Das Aus-der-Haut-Ziehen oder die subcutane Methode von 1830 an. Diese ist wohl in dem Bestreben, eine für das Muttertier weniger gefährliche Operation zu erzielen, ausgebaut. Hierzu sind nur wenige Instrumente nötig. Außerdem ist diese Methode auch mit weit geringeren Gefahren intrauterin möglich. Sie ist mühsam, aber durchaus brauchbar und wird noch in neueren Lehrbüchern als gangbar bezeichnet.

III. Etappe: Von 1880 bis auf unsere Zeit. Sie ist gekennzeichnet durch das Arbeiten mit sägenden Instrumenten, das viele neue Möglichkeiten erlaubt. Diese letzte Etappe ist zu bekannt und historisch noch zu jung, als daß sie gewertet werden kann und soll.

Selbstverständlich lassen sich die einzelnen Etappen nicht so genau trennen, wie schematisierend dargelegt wurde. Ineinandergreifen der einzelnen Etappen, Rückfälle und vorauseilende Einzelfälle kommen so gut wie bei jeder anderen historischen Unterteilung vor.

\section{Etappe}

Über die Tiergeburtshilfe im Altertum ist nur wenig überliefert. Embryotomische Eingriffe erwähnen nach Baranski (1886) einzig Absyrtus (300-360 nach Christus) und Columella (etwa 40 nach Christus).

Absyrtus schreibt: «Man soll auch die Hand in die Geschlechtstheile und in die Gebärmutter der Stute hineinführen, und das Fohlen, wenn es schon behaart ist, beim Maul fassen, es zu würgen und den Kopf zerquetschen. Wenn es aber im Mutterleibe lebt, so zerreiße zuvor die das Fohlen umschließenden Membranen.»

Columella aber äußert sich schon genauer, wenn er sagt: «Man muß außerdem auf jenes Thier, welches nahe der Geburt ist, Acht geben, nicht 
anders als es die Geburtshelfer zu thun pflegen, da das Schaf auf eine gleiche Weise, wie ein Weib gebärt, und auch häufig muß sie jeder Hilfe bar mit Mühe gebären. Deshalb soll der Schäfer in der Thiermedicin bewandert sein, damit, wenn es der Fall erheischt, er entweder die ganze Geburt herausnehme, wenn sie in den Geschlechtstheilen quer liegt, oder mit dem Messer zerschneiden ohne die Mutter zu beschädigen, und stückweise herausnehmen, was man bei den Griechen ,Herausnahme der todten Geburt " nannte.»

Aus diesem letzten Satz aber geht hervor, daß diese Operation auch den Griechen bekannt war. Weiteres darüber ist uns persönlich nicht bekannt. Um 1320 nach Christus soll ein Araber, ABU BeKr IBN Bedr zur Geburtshilfeleistung bei toten Fohlen mit einem kleinen Messer zwischen den Fingern in die Geburtswege eingegangen sein, und Haken an den Augenhöhlen, Kinnwinkeln und Halswurzeln angebracht haben (Rieck).

1545 hat Colerus in einem lange Zeit neuaufgelegten Werk über Zucht und Krankheiten der Haustiere ähnlich Columella geschrieben: «Wann ein Schaf gebieret, so muß ein Schäfer dabey seyn, und dem gebährendem Schafe helfen, wo ihm Hülfe von Nöthen ist, daß es daßlebige gantz oder in stückigt von sich bringe.»

1673 berichtet Michael Harward in The Herdmans mate ... über ein Fingermesser, das er zur Embryotomie gebrauche (de Bruin).

Diese spärlichen Angaben, die ohne genaue Beschreibung der Methode geblieben sind, werden verständlich, wenn wir uns den Zustand der Tiergeburtskunde zu jenen Zeiten vergegenwärtigen: rohe Empirie von Hirten und alten Weibern! Noch 1818 schreibt der bedeutende Jörg: «Schäfer und Hirten, bey welchen man sich gewöhnlich, wenigstens in unseren Gegenden, Raths erholt, wenn ein Hausthier eine schwere Geburt erleidet, muß nicht allein die Geburtshilfe, sondern die ganze Quacksalberey, die sie an Thieren nur zu oft vornehmen, untersagt werden. Es ist eine Classe von Menschen, die für ein solches Fach zu wenig Bildung haben, zu voll von Vorurtheilen ist und besseren Rath sehr schwer oder gar nicht annimmt. Auch sind ihre Hände zu derb und hart, als daß sie sich für ein solches eignen sollten.»

Wenn von nun an die Meldungen häufiger und klarer werden, so ist das zum großen Teil dem Umstande zu verdanken, daß durch die Gründung der ersten Tierarzneischule zu Lyon im Jahre 1763 der tierärztliche Bildungsgedanke rasch gefördert wurde und die Tiermedizin zu wissenschaftlichem Ansehen kam. Führend nahmen sich einige Menschenärzte dem jungen Zweig der Medizin an. So ist auch die folgende Entwicklung zu verstehen: eine weitgehende Anlehnung der tierärztlichen Geburtshilfe an die men- 
schenärztliche, ein Spielen mit theoretischen Möglichkeiten von Männern, die zum Teil nur wenig oder gar nicht mit der tierärztlichen Praxis verbunden waren.

Ferner ist zu bedenken, daß auch in der Landwirtschaft ein Umschwung sich fühlbar machte. Neue Methoden traten an Stelle der altüberlieferten.

Auch mag durch die großen Seuchenzüge der Wert des einzelnen Tieres gestiegen sein.

Es ist noch einer Erfindung der menschenärztlichen Geburtshilfe zu gedenken, die von nun an für einige Zeit in der theoretischen Tiergeburtshilfe eine große Rolle erlangte: der Geburtszange.

Wir möchten hier auf den Prioritätsstreit, ob der Familie der ChamberLENS oder PALFyns (1650-1730) die Erfinderehre gebührt, nicht eintreten und verweisen auf die einschlägige humanmedizinische Literatur. Tatsache ist aber, daß die Zange erst mit Palfyn, 1723, größere Bedeutung erlangte. DiePGen spricht von der «wichtigsten Errungenschaft der geburtshilflichen Praxis » im Zeitalter des Barocks, und F. C. Müllen schreibt, daß die humane Geburtshülfe des 19. Jahrhunderts in zwei Hauptschulen unterteilt werden könne, in Osianders Entbindungskunst und Boers Geburtshilfe.

Bei Friedrich Benjamin Osiander (1759-1822), Professor in Göttingen, gehörte der Gebrauch der Geburtszange zum Gewöhnlichen. So sollen $54 \%$ der Geburten Zangengeburten gewesen sein, während sein Gegenspieler Lukas Johann Boer (1751-1835) in Wien nur 0,6\% der Frauen mit der Zange entband.

In Hermann Vitet (1763-1809), ursprünglich Menschenarzt, war der Veterinärmedizin ein warmer Befürworter der Geburtszangen beschieden. Schreibt er doch: «Die Viehärzte, die jede auch noch so nützliche Entdeckung verachten, gebrauchen lieber das Seil, als die Zange.»

«Die Zange besteht aus zwey Armen, deren Löffel ausgeschnitten und gebogen sind, so daß sie sich an der Rundung des Kopfes des Foetus anlegen können. Man kann jeden Arm einzeln einbringen und nachher beide Arme in dem Schlosse fest miteinander verbinden. Die Arme müssen von dem Löffel an bis zu der Axe stark, lang, ein wenig platt und der Stiel breit und dick seyn ... Wenn nun die Löffel der Zange die Seiten- und hinteren Theile des Kopfes eingeschlossen halten, so vereinigt man beide Arme der Zange in dem Schlosse miteinander und zieht sie mit allmählig zunehmender Gewalt in Viertel Windungen um den Kopf besser zusammen zu drücken, länger zu machen und nach der Öffnung des Beckens zu bilden an ... Die Zange hat auch den Nutzen, daß sie die Scheide erweitere.» 
Wir sehen daraus, daß Vitet die Zange nicht nur zur Extraktion, sondern auch zur Volumenverkleinerung des Föten gebrauchte. Er trachtete damit den Umfang des fötalen Kopfes zu verkleinern. Erst wenn das ungenügend ist, empfiehlt er den Gebrauch des Bistouris. Dann aber kommt nochmals die Zange zu Ehren, nämlich zum Herausziehen der zerstückelten Teile. Bevor wir auf die einzelnen von ihm empfohlenen Operationen eingehen, möchten wir die Indikationen, die Vitet zur Zerstückelung veranlassen, erwähnen: Fehlerhafte Bildung der Mutter dadurch, daß die hinteren Hüftbeinäste zu nah beieinander sind und andererseits übermäßige Größe des Jungen, wobei entweder der Kopf oder die Brust zu groß sein kann. Also noch recht unbestimmte Indikationen. Als Hilfsmittel hierzu zählt er die Zange und das Skalpell oder krumme Bistouri, welches « an dem obern Ende an einer kleinen Stelle gekrümmt und an der convexen Seite stumpf, an der hohlen Seite aber scharf» sein muß und «so lang seyn soll als die Scheide».

An Operationen empfiehlt er das Wegschneiden des Kopfes: «dann setzt man die Schneide hinter dem rechten Ohre des Foetus an und schneidet den größten Theil des Hirnschädels weg, macht hierauf den nämlichen Schnitt auch von dem linken Ohre an, und schneidet so den übrigen Theil des Kopfes weg und verfährt nachher ebenso mit den übrigen Theilen des Körpers.»

Statt den Kopf abzutragen, kann man aber ihn auch nur mit dem Skalpelle öffnen und ihn nachher mit der Zange zusammendrücken.

Ferner ist noch die Verkleinerung des Brustumfanges näher beschrieben: «In Fällen der Art durchschneide man also mit einem krummen Skalpelle die Rippen an beiden Seiten, drücke mit der Hand oder mit der Zange die Brust zusammen, und ziehe dann den Foetus beym Kopf heraus.»

Über die oben angegebene weitere Zerstückelung des Jungen äußert er sich nirgendswo noch genauer, schreibt aber: «Diese Operation hat doch große Schwierigkeiten, die erste ist, das Instrument in die Gebärmutter zu bringen, und den Foeten in Stücken zu zerschneiden, ohne die Gebärmutter oder die Scheide zu verletzen; und die zweyte: grade den Foetus in solche Stücke zu zerschneiden, als durch die äußere Öffnung des kleinen Beckens durchgebracht werden können.»

Diese Operationen sind also noch recht ungenügend und stark theoretisch. Sie lassen aber doch deutlich das Suchen nach einer allgemeingültigen Methode erkennen, und das ist ein wesentlicher Fortschritt.

Von weiteren geburtshilflichen Operationen dieser Epoche berichten 1778 Joh. Adam Kersting (1726-1784), der hierzu ein dem Roederschen Fingerbistourigleichendes Ringmesser konstruierte(Rieck), und 1793 JоH.GÜNTHER 
Eberhard, Arzt zu Zeist, der eine Methode der Embryotomie angegeben haben soll, die uns persönlich aber nicht bekannt ist (de Bruin).

1806 hat Fromage de Feugré (1770-1812) in dem Dictionnaire de l'abbé Rozier (1734-1793) von dem wiederholten Wegnehmen von Vorderfüßen bei emphysematösen Kälbern durch gewaltsamen Zug berichtet, und in demselben Jahr meldet BlankaIN (?-?), dieselbe Operation ausgeführt zu haben, mit Hilfe eines Spatels und eines Fingermessers.

Ebenfalls 1806 sollen die Engländer KnIP Vater und Sohn das Aus-derHaut-Ziehen der Gliedmaßen geübt haben, indem sie mit der Hand durch ein Loch im Stall, wodurch der Urin abfließt, eingegangen seien und die Gliedmaßen von im Stallinnern liegenden toten Kälbern subcutan ausgezogen hätten (de Bruin). Diese Angaben näher zu überprüfen, war uns leider nicht möglich.

Über die Wegnahme von Kopf und Vorderfüßen durch den Engländer SkELletT im Jahre 1811 haben wir schon durch den ungläubigen Ausspruch Joergs gehört.

Im Schweizer Archiv für Thierheilkunde 1816 berichtet Tierarzt JoHann $\mathrm{J}_{\text {Аков }}$ SchlumpF über die damals viel diskutierten Speckkälber und schreibt: «Vermuthung ist, das Versehen als Ursache dieser Erscheinung anzunehmen ... Vorzüglich soll es der Dogghund sein, an dem sich die trächtige Kuh versieht.» «Jede Kuh brachte ein Speckkalb, das aus dem Uterus geschnitten werden mußte.»

Wie aus diesem knappen Literaturüberblick ersichtlich wird, ist doch gegen Ende der ersten Etappe ein Ansteigen der Meldungen über Zerstückelung und ein Suchen nach dem besten Weg ersichtlich.

$\mathrm{Da} 3$ diese aber doch nur Einzelfälle geblieben sind, denen sich die große Menge verschloß, ja sogar ungläubig gegenüberstand, beweisen die Äußerungen Vitets, der sich beklagt, daß die Viehärzte jede noch so nützliche neue Entdeckung verachteten, und des Professors der Entbindungskunst Jörg, der von einer Lüge, die nicht die erste sei, spricht.

\section{Etappe}

Im Verlaufe dieser Etappe entwickelte sich die Embryotomie zu einem festgefügten Ganzen, das in zunehmendem Maße auch vom Landtierarzt ausgeübt wurde. Ermöglicht wurde dieser Fortschritt durch den Einfluß der tierärztlichen Lehranstalten auf die tiermedizinische Forschung und dadurch, daß an Stelle der vordem meist ungebildeten, sozial tiefstehenden Tierheil- 
kundigen nun mit zunehmender Zeit immer besser naturwissenschaftlich geschulte Tierärzte traten, deren Wirken auch in der Öffentlichkeit und bei Vertretern anderer Wissenschaften Anerkennung fand. An den Schulen wurden unter anderem auch über Anatomie, Physiologie und Pathologie des Geburtsvorganges gelehrt und geforscht. Einen für die Festlegung der Indikationen zur Embryotomie wichtigen Fortschritt bildete die scharfe Fassung des Geburtshindernisses von Seiten des Föten in die Termini technici der Lage, Stellung und Haltung an Stelle der früheren langen und meist unklaren Umschreibungen. Verdient darum haben sich vor allem Harms (1867) und FrancK (1876) gemacht.

In die Wende zu dieser Etappe fällt das Wirken des Leipziger Ordinarius für Geburtshilfe beim Menschen, Johann Christian Gottfried Jörg (1779-1856). Bedeutungsvoll für sein geburtshilfliches Handeln war ein Aufenthalt bei Boer in Wien. Jörg verfaßte neben geburtshilflichen auch zahlreiche Arbeiten über andere Gebiete der Humanmedizin. Es ist interessant, daß im Biographischen Lexikon berühmter Ärzte aller Zeiten ... von A. Hirsch, 2. Auflage, Wien, Jörgs bedeutungsvolles Werk über die Tiergeburtshilfe keine Erwähnung findet.

«Wer aber nur einigemale bey schweren Geburten der Thiere geholfen hat», schreibt Jörg, «und besonders mit seiner Hand in die Gebärmutter eingegangen ist, wer das unaufhörliche Pressen und unruhige Verhalten der Gebärmutter dabey aus der Erfahrung kennt, der wird wohl davon überzeugt seyn, daß das Hanthieren mit einem Messer in der Gebärmutter ohne Verletzung des Mutterthieres unmöglich sey. Vorteilhafter ist es in solchen desparaten Fällen für den Ökonomen, anstatt die fast unmögliche Zerstückelung des Jungen vorzunehmen, das alte Gebärende zur rechten Zeit zu schlachten.»

Es mag auf ersten Anhieb verwundern, daß wir ungeachtet dieses ablehnenden Standpunktes das Wirken Jörgs als Wendepunkt genommen haben. Wir möchten aber unsere Embryotomiegeschichte nicht unabhängig, sondern vielmehr in enger Wechselbeziehung zur Gesamtentwicklung der Geburtshilfe gestellt wissen. Unter diesem Gesichtspunkt betrachtet, wird unsere Einteilung verständlich.

Jörg hat 1808 unseres Wissens als erster ein Werk, das ausschließlich die Tiergeburtshilfe abhandelt, veröffentlicht und dieses durch einen Tafelband vervollständigt.

Die Hebung der Tiergeburtshilfe und die Ausbildung tüchtiger Geburtshelfer wollte er mit seinem Werk fördern: «Es gilt dies nur von der Ent- 
bindungskunst oder der Geburtshilfe des Menschen und nicht von der der Thiere, welche letztere bey weitem noch nicht so beachtet und bearbeitet ist, als sie es verdiente. Auch hat man die Geburtshilfe der Thiere noch nicht als einen für sich bestehenden Theil der Thier-Heilkunde, schriftlich wenigstens noch nicht, behandelt, und ohne Zweifel gehört diesem Umstand ein groBer Theil der Unvollkommenheit zu, in welcher sich dieselbe noch befindet.»

Die Förderung, die von Jörg ausging, wird besonders deutlich, wenn wir uns das Ansteigen der tier- und embrytomiegeburtshilflichen Arbeiten, insbesondere der Lehrbücher, die ja der Ausbildung des Tierarztes am unmittelbarsten dienen, vergegenwärtigen (siehe Literaturverzeichnis). Jörgs wird von mehreren dieser späteren Autoren ehrend und lobend gedacht.

Was nun das Werk selber anbetrifft, so ist es, im Rahmen seiner Zeit betrachtet, ein vorzügliches, wissenschaftlich gehaltenes Buch, das noch heute, fast hundertfünfzig Jahre später, lesenswert ist.

Er teilt den Stoff in einen theoretischen Teil, worin er die Anatomie, Physiologie und Pathologie der Mutter und des Föten behandelt, und in einen praktischen Teil ein. Wir müssen es uns versagen, in diesem Zusammenhang näher darauf einzugehen.

Jörg, von F. C. Müller als «einer der erfolgreichsten Vertreter der Wiener Schule» (Boer) genannt, «übte durch seine Lehrtätigkeit in Leipzig einen großen segensreichen Einfluß aus». In der Humanmedizin zu der Richtung Boers neigend, gehörte er in der Tiermedizin zu den begeisterten Anhängern der Geburtszangen. Ja, er hat sỏgar in Anlehnung an die von Boer für menschenärztliche Zwecke geschaffene Zange speziell eine für veterinärmedizinische Verhältnisse konstruiert. Wir wollen an dieser Stelle nicht näher auf die interessante Entwicklung der Zangengeburtshilfe in der Veterinärmedizin eintreten, möchten aber doch noch erwähnen, daß er im Gegensatz zu Vitet nirgends die Zerkleinerung des Föten mittels der Zange empfiehlt, sondern diese nur zur Extraktion verwendet, gleich wie Boer in der Menschengeburtshilfe.

«Wenn hier von einer Geburtszange die Rede ist, so wird kein Instrument darunter verstanden, wodurch das junge Thier im Mutterleibe getödtet oder stark verwundet und so hervorgezogen wird, sondern wir begreifen darunter ein Instrument, welches den Kopf des Jungen (denn nur für diesen ist sie gebaut) behutsam und auf eine unschädliche Weise faßt, und denselben unbeachtet des Lebens des Jungens hervorbewegt.»

Als ausführbar betrachtet er einzig die Zerkleinerung des wassersüchtigen Föten mittelst Troicar oder kleinem Messer. «Der Troicar wird in der 
hohlen Hand in die Gebärmutter eingeführt und dort ohne Umstände in die mit Wasser angefüllte Stelle eingetrieben.»

Sonst aber kommt einzig die Ausziehung mittelst Zange, scharfem Haken, Band oder Seil, oder aber der Kaiserschnitt oder die Schlachtung in Frage.

Im folgenden lassen sich nun zwei hauptsächliche Richtungen unterscheiden, wobei die mehr der Amputation zugetane Richtung vor allem in die Zeit bis 1830 und die das Aus-der-Haut-Ziehen bevorzugende vorwiegend von 1830 an datierbar ist.

\section{A. Die Zeit der Amputationen}

Hierunter fallen vor allem die Amputation des Kopfes, der Gliedmaßen und die noch eingehender abzuhandelnde operative Geburtshilfe bei der hundesitzigen Stellung.

Werfen wir zunächst einen Blick auf einige in dieser Zeit neugeschaffene Instrumente, um dann die einzelnen Operationen und ihre Indikationen zu erläutern.

\section{Instrumente}

1823 schnellwindige Schraubenzange von Fey.

1823 Hakenmesser von Fey.

1830 Große Zahnzange von Binz.

1830 Kleine Zahnzange von Binz.

1830 Krummes Fingermesser von Günther.

1830 Großes Scherenmesser von Günther.

Die Feysche schnellwindige Schraubenzange besteht aus einer runden Scheide, in der zwei über einer Schraube laufende Zangenschenkel mit je zwei Zacken sind. Sie diente zur Herausnahme losgetrennter oder gelockerter Teile.

Das schnellwindige Hakenmesser von Fey besteht aus einem an einer Schraube in einer Scheide laufenden Hakenmesser und wurde zur Zerstükkelung allzugroßer Kälber, Molen und Mißgeburten gebraucht. Binz empfiehlt « dieses zur Zerstückelung vorzügliche Instrument» bestens.

Die große Zahnzange von Binz, «welche beim Zerstückeln junger Thiere im Mutterleibe mit großem Nutzen gebraucht werden kann, ist ein Instrument, welches aus zwei löffelähnlichen, eisernen Kapseln und zwei Armen besteht, in welchen sechs Zähne an zwei in einer Rinne laufenden eisernen Stangen befestiget und verborgen liegen ...» Die Anwendung der Zange ge- 
schieht auf folgende Weise: «... faßt alsdann mit beiden Händen diesselbe ... und bringt sie spielend in die Gebärmutter hinein. Jetzt sucht man den Theil des Körpers der herausgezogen werden soll (welcher aber jedesmal zuvor mehr oder weniger losgetrennt seyn muß) zwischen die beiden Blätter der Zange zu bringen, welche dann sogleich mit der Kapsel geschlossen werden kann. Ist dieses fertig, so läßt man durch einen Gehilfen an beyden Handgriffen der eisernen Stangen anziehen, inde $\beta$ der Geburtshelfer diese Zange nach einwärts drückt und festhält, wodurch dann die Haken aus ihrer Scheide hervorkommen, in den Theil eingreifen und festhalten, so, daß das angefaßte Stück herausgezogen werden kann.»

«Die kleine Zahnzange kann beim Zerstückeln des Kopfes und einer Mola mit Nutzen zu Hilfe gezogen werden, und erleichtert dem Geburtshelfer das Herausnehmen der losgetrennten Theile» (Binz).

Das krumme Fingermesser nach Günther, «ist eine einfache, 21/2 Zoll lange Klinge, die in der Form so ziemlich der Klinge eines Rebenmessers entspricht, und zwei feststehende Ringe auf dem Rücken hat.» (Harms)

Das verdeckte Messer nach Günther, «besteht aus einer zweischneidigen Klinge, die ca. $2 \frac{1}{2}$ Zoll lang und so mit dem Hefte verbunden ist, daß sie beliebig vor- und rückwärts geschoben werden kann» (Harms).

Es ist auffallend, daß hauptsächlich deutsche Geburtshelfer sich im Konstruieren von schneidenden Instrumenten und Geburtszangen hervortaten, während ihre französischen Kollegen sich mit viel einfacheren Werkzeugen begnügten ${ }^{1}$. Keines dieser Instrumente, mit Ausnahme des Güntherschen krummen und verdeckten Messers, konnten sich behaupten. Kompliziertheit, Kostspieligkeit und nur begrenzte Anwendungsmöglichkeit wurde ihnen von späteren Geburtshelfern vorgeworfen.

\section{Indikationen}

Die Embryotomie bildet zu jenen Zeiten immer eine ultima ratio, denn die Gefahren und Anstrengungen für Muttertier und Geburtshelfer sind beträchtliche.

1 So erwähnt Hurtrel D'Arboval (1777-1839) in seinem Dictionnaire de médecine et chirurgie vétérinaire die komplizierten Feyschen Instrumente nicht, während sein Übersetzer RENNER (1779-1850) es für angebracht findet, eine lobende Anmerkung darüber hinzusetzen: «Die thierärztliche geburtshülfliche Praxis hat einebedeutende Erweiterung durch die Erfindung neuer Instrumente zum Behufe der Zerstückelung des Jungen und ihre verschiedene Anwendung von Seiten des braven Schweizerischen Thierarztes Sebastian Fey erhalten.» 
So hält Jörg diese Operation für unmöglich, Binz glaubt sogar, daß «das gewaltsame Trennen der Beckenknochen der Mutter durch Zug am Jungen » «mit Vorsicht und der gehörigen Sorgfalt verrichtet ... meistens keine so gefährlichen Folgen hat, als wenn der Bauch- oder Flankenschnitt, oder das Zerstückeln des Jungen vorgenommen wird.» J. H. Fr. GüNTHER (1791 bis 1859) schreibt ebenfalls 1830: «Diese letzte Nothülfe opfert das Junge und setzt die Mutter einer größern oder geringern Gefahr aus.» Dennoch empfiehlt er aber: «Man entschließe sich deshalb zuletzt dazu, verschiebe jedoch selbige nicht, wenn man die feste Überzeugung gewonnen, daß die Geburt auf andere Weise unmöglich ist.» Hurtrel D'Arboval: «Übrigens hat diese Operation immer große Schwierigkeiten; die erste besteht in der richtigen Einführung des Instrumentes in den Uterus, die zweyte darin, daß die verschiedenen Theile des Foetus zerschnitten werden müssen, ohne die Bärmutter und Scheide zu verletzen. Im allgemeinen dauert eine solche Operation lang, und ist für den Veterinärarzt eben so ermüdend, als für die Mutter, welcher man von Zeit zu Zeit Ruhe gestatten muß, angreifend.»

Diese Beispiele ließen sich noch beliebig vermehren. Die Urteile werden erst mit fortschreitender Entwicklung der subcutanen Methode günstiger (siehe dort).

Wir finden zunächst noch recht unpräzise Indikationen, so empfiehlt Fey die Embryotomie bei allzugroßen Kälbern, wobei darunter verstanden werden, «solche die größer sind (auch regelwidrig größer) als der Durchmesser des Beckens ist. Bald ist nur der Kopf, der Hals, und bald auch der ganze Rumpf mit zu groß», bei Speckkälbern, Wasser-, Luft-, Dunst- oder Blastkälbern, Kälbern mit abnormen Gewächsen und bei Molen.

Binz, Günther, Huvellier kennen im wesentlichen dieselben Indikationen, nur mit dem Unterschied, daß sie diese etwas genauer definieren.

So übt z. B. Günther die Embryotomie aus, «wenn das Junge absolut zu groß, oder aber das Becken absolut zu klein ist», und als spezielle Fälle führt er an - immer als ultima ratio verstanden -: «ist der Schenkel im Knie gebogen», «liegt der Schenkel schlank unter dem Leibe verschlagen», "wenn der Kopf richtig innesteht, beide Vorderschenkel aber zurück sind», "wenn beide Vorderschenkel richtig innestehen und der Kopf mit dem Maule nach der Brust, das Genick aber gegen den Muttermund steht», "wenn bei richtig innestehenden Vorderschenkeln der Kopf nach der rechten oder der linken Schulter hin verschlagen ist», "wenn der fehlende Schenkel im Sprunggelenk gebogen ist », «wenn das Junge in Rückenlage mit dem Vordertheil eintritt», "wenn bei Rückenlage des Jungen die Sprunggelenke 
eintreten », «wenn bei Rückenlage das Junge mit der Kruppe eintritt », «wo Vorder- und Hintertheil zugleich eintritt», "wenn das Junge ohne Schenkel und Kopf mit der Seitenfläche des Körpers oder dem Rücken vor die Geburt tritt », «bei Mißgeburten », «wenn das Junge ganz oder theilweise ohne Gelenke ist», "Speckkälber, Dunstkälber und wassersüchtige Zustände (Wasserkopf, Bauchwassersucht, Hautwassersucht), Mole oder Mondkalb ».

Diese Indikationen werden im Laufe der Zeit immer schärfer gefaßt und besser klassiert. So unterteilt z. B. 1875 SAINT-CyR die im wesentlichen gleichen Indikationen in zwei große Gruppen :

\section{Infolge Beckendeformitäten der Mutter, oder häufiger}

2. das Hindernis liegt beim Föten selbst infolge zu großen Volumens, emphysematösen Föten, Hydrocephalus, Ascites, Gliedmaßen oder Halskontraktur, Mißbildungen, gewissen fehlerhaften Stellungen.

\section{Die einzelnen Amputationen}

Unter den vielen Schriftstellern, die die Amputation erwähnen, können wir nur einige nennen. Vorwiegend ausgeführt wurden die Amputationen bis ungefähr 1830 , weil man vorher die weniger gefährliche subcutane Methode noch nicht kannte. Daß sie aber auch noch nach dieser Zeit empfohlen und von einigen Schriftstellern sogar als Methode der Wahl angepriesen wurde, mögen die Arbeiten des französischen Landtierarztes Donnarieix, der die Embryotomie sehr gefördert hat, beweisen.

Im Prinzip ist die Methode immer dieselbe: Absetzen einer Extremität in einem Gelenk (Ellenbogen-, Knie-, oder Hüftgelenk) oder Durchtrennen der Synsarkosemuskeln mit irgendeinem der vielen schneidenden Instrumente und Ausreißen von Hand oder mit Zangen, falls die Verbindungen nicht genügend getrennt werden konnten. Abtrennen des Kopfes entweder im Kopfatlasgelenk oder aber an der Halswirbelsäule, oder Durchtrennen der Kopfnähte und dadurch Verkleinerung des Kopfumfanges, Evisceration der Baucheingeweide von der Bauchhöhle oder von der Brusthöhle aus durch das Zwerchfell hindurch, der Brusteingeweide durch Durchschneiden der Rippen oder durch Eingehen zwischen den ersten zwei Rippen.

Im folgenden soll nun noch anhand einiger Zitate belegt werden, wie einzelne Autoren vorgegangen sind.

Sebastian Fey «ziehet» bei der Zerstückelung allzugroßer Kälber, «die vorderen Gliedmaßen, welche man zuerst herausoperieren will, hervor, schlauft beide Füße, jeden mit einem Schlaufenband zwischen dem Fessel- 
und dem Kronengelenk gut an ... nimmt dann das krumme Hakenmesser... fährt so mit dem Messer in der Hand durch die Geburtswege durch, bis auf die Schulter des Jungen, leitet mit dem Zeigefinger die Spitze des Messers, und drückt selbe oben am Ende des Schulterblattes durch die Haut und fleischigsten Theile und fängt an die Haut im ganzen Umkreise derselben so weit möglich - loszutrennen ... kann man nicht mehr weiterschneiden, und ist die Schulter noch nicht ganz losgetrennt, so wird das Messer auf die Seite gelegt, die Zange ... ergriffen ... läßt durch den gleichen Gehilfen durch Vorwärtstreiben der benannten Scheide recht gut schließen, dann an dem Ringe a nach Erfordernis ziehen, links und rechts umdrehen wodurch die Schulter ganz los wird und ausgezogen werden kann.» Bei Speckkälbern setzt er den Kopf am Halse mittels der schnellwindigen Zange ab, amputiert dann auf obige Art die Gliedmaßen, durchschneidet die Bauchnaht und evisceriert die Eingeweide. Wasserkälber geht er durch Anstechen des wasserhaltigen Teiles mit dem Messer an.

BıNZ, der in seinem Lehrbuch nur die Amputation angibt (1830), löst die Füße im Kniegelenk ab, und öffnet die Brust und Bauchhöhle. Die Zerstückelung des Kopfes rät er nur im äußersten Fall vorzunehmen. «Das Schwierigste, das Beschwerlichste und Gefährlichste ist das Zerstückeln des Kopfes; es erfordert alle Vorsicht und Geschicklichkeit im Operieren und gut eingerichtete Instrumente. Ich entblößte daher meinen rechten Arm, überzog ihn mit Öl, nahm ein in der Hand verborgen gehaltenes, geballtes, an der Spitze mit einem Köpfchen versehenes Bistouri und suchte mit demselben in das Maul des Jungen einzukommen, um die Back- und Kaumuskeln durchzuschneiden ... Als diese beidseits soweit durchschnitten waren, daß nur noch einige Stellen festhielten, welche ich aber nicht mehr in Anschlag brachte, legte ich ein Seil an dessen Unterkiefer und ließ mit Gewalt ausziehen, wodurch es mir auch glückte, denselben von Oberkiefer loszureißen und herauszubefördern. Nun versuchte ich die Verbindung des Hinterhauptbeins mit dem ersten Halswirbelbeine zu trennen ... und als dieses geschehen war, fesselte ich denselben an, und ließ mit Gewalt ausziehen, worauf sodann der Kopf vom Rumpfe abgerissen und herausbefördert wurde.» Ein anderes ebenso schwieriges Verfahren: «Nun faßt der Operateur das Messer und geht mit demselben in der Hand verborgen gehalten in die Gebärmutter ein, und schneidet dann die obere Kopfwandung in ihren Nähten durch. Ist dies geschehen, so müssen mit der kleinen Zange die abgetrennten Stücke, eines nach dem andern, herausgenommen werden.» 
Günther empfiehlt neben der Amputation ebenfalls die subcutane Methode, während 1831 Hurtrel d'Arboval die ein Jahr zuvor von Huvellier ausgeführte subcutane Methode verwirft und nur die Amputation empfiehlt.

Die Amputationen finden wir in allen Lehrbüchern angegeben, wobei aber mit fortschreitender Zeit der subcutanen Methode immer mehr Gewicht beigelegt wird: Günther 1830, Baumeister 1844, Baumeister-Rueff 1853, Baumeister-Rueff 1869, Rainard 1850, Dieterich 1845, Harms 1867, Saint-Cyr 1875, Franck 1876, Saint-Cyr und Violet 1888, Deneubourg 1880.

Auch in den tierärztlichen Zeitschriften und in den Fachgesellschaften (Société centrale de médecine vétérinaire) spiegelt sich das lebhafte Interesse wider, das man der Embryotomie entgegenbrachte: Cannu 1837, Delafond 1849, Hübner 1847, Mignon 1849, Margraff 1857, Donnarieix 1857, Aubry 1859, Palat 1861, Gierer 1867 und 1871, Recordon 1877 usw.

Aber auch das gewaltsame Ausreißen der Gliedmaßen wurde zu jenen Zeiten noch vereinzelt geübt. So berichtet RaInARD (1850), daß Texier mehrmals Gliedmaßen ausgerissen habe, um nicht mit dem schneidenden Instrument in die Gebärmutter eingehen zu müssen, daß aber die Mehrzahl der neueren Geburtshelfer sich vor der Amputation nicht mehr scheuten. 1842 beschreibt Drouard das Ausreißen der Hintergliedmaßen bei einem emphysematösen Föten. «Un fort crochet, implanté du coté droit dans les muscles qui recouvrent l'articulation coxo-fémorale; six à sept personnes tirent dessus, tandis que d'autres soutiennent la jument et bientôt la peau et les muscles se déchirent, le fémure est désarticulé et le membre tiré au dehors.»

Von besonderem Interesse ist noch die Operation bei der hundesitzigen Stellung. CANNU, der bereits 1837 die Bauchvertikallage mit der hundesitzigen Stellung verglich, hatte dabei eine Methode entwickelt, die auch heute noch angewandt wird. Er hat nach Franck (1876) bei Fohlen zuerst die Brust aufgeschnitten und exenteriert, dann die Wirbelsäule durchtrennt und die Wendung des Hinterteiles vorgenommen und nachher extrahiert.

Über diese Operation entspann sich in französischen Zeitschriften (unter anderem im Recueil de médecine vétérinaire) und in der Société centrale de médecine vétérinaire ein Streit.

Nachdem Donnarieix zuerst (1858) geschrieben hatte: «Lorsque dans le cas de présentation antérieure tout l'avant-train du poulain étant sorti hors de la vulve, l'arriere-train ne peut venir, parce que les deux membres postérieures s'arc-boutent contre le plancher du bassin, il faut, dans ce cas, 
éventrer le poulain pour vider l'abdomen, et alors essayer d'allonger les membres en arriere. Si on ne peut y réussir, il faut faire la section du cadavre au niveau des reins, faire culbuter dans la matrice le tronçon qui reste, et opérer son extraction par le derrière en déployant les membres postérieurs 》, krebst er 1859 wegen der Kritik einiger Tierärzte zurück. Er entschuldigt sich, der Text habe wegen seiner Kürze zu Unklarheiten Anlaß gegeben. «Telle n'était pas ma pensée, telle aussi ne devait elle pas être. Cette erreur, encore une fois, résulte évidémment de la trop grande concision ou laconisme, que j'ai mis dans le détail des faits: Car la répulsion des membres postérieures dans la matrice n'a jamais été pour mois chose possible. En second lieu, si dans un cas j'ai fait l'amputation du tronc au niveau des reins, c'était pour être moins géné par la masse pendant hors de la vulve. Quant à renverser du côté de la matrice la portion du tronc réstante, cette version ne m'a pas été possible, pas plus que dans le premier cas, l'éspace si oppose.» Donnarieix schlägt nun folgendes Verfahren vor: "Pour en finir par un seul mot, pour que l'accouchement s'effectue, le membre doit être allongé parallement au parois du ventre pour détruire l'angle formé par le jambe et le canon, et tout se résume ici: $1^{\text {er }}$ Eventrer le poulain et retirer les entrailles, $2^{\text {e }}$ Saisir les deux paturons, isolément et successivement dans l'anse du corde, et opérer au moyen d'aides, une traction isolée et successive sur chaque membre, pour en obtenir l'allongement au dehors.»

Ein Jahr später beschreibt er nochmals seine Methode und sucht diese durch eine geometrische Figur zu verdeutlichen (1859).

Inzwischen waren aber einige Mitteilungen anderer französischer Tierärzte erschienen, die die von Cannu angegebene und von Donnarieix zuerst als gangbar bezeichnete, dann aber widerrufene Operationsart als durchführbar erklärten. So unter anderen von M. E. Rochard, der über Donnarieix schreibt: «qu'il a en tort de se retracter plus tard», und von Cannu selbst: «que je suis loin de penser comme luis. Depuis trente ans, ce cas s'est présenté a moi peut être douze fois, et presque toujours, le moyen qu'il traite d'irrationnel et inéxécutable a été le seul qui m'a servi à sauver la mère. Faire la section du cadavre au milieu des reins, refouler la croupe dans la matrice, luis imprimer un mouvement de bascule, saisir les membres postérieures et en faire l'extraction.» (1860).

Gleich wie Cannu gingen vor: Cartwright 1846, Marty 1858, Rossignol, Rochard, Walter 1861, Obich 1868, Harms 1867, Franck 1876 u. a.m.

Harms und FrancK bemerken jedoch, daß « diese Wendung übrigens ein sehr schwieriges Stück Arbeit und durchaus nicht ohne Gefahr für das Mut- 
terthier» sei (Franck). Harms empfiehlt deshalb auch auf die Wendung zu verzichten und lieber die beiden Darmbeine subcutan zu entfernen.

Über eine andere Methode berichtet 1844. Dieterich, «der offenbar jedoch die Lage nicht selbst gesehen» (Franck) hat. «Ist der Kopf und die Brust aus dem Wurfe gezogen, widerstehen aber die Hinterfüße noch, so kann man neben dem eingezwängten Fötus mit der Hand nicht eingehen; auch in diesem Fall entleere man die Brust- und Bauchhöhle gänzlich durch die vordere Brustöffnung, fühle nun genau durch die Bauchwandungen des Fötus nach den vorderen Theilen der Hinterfüße, und wenn man sie weder von hier aus noch durch Gegenwirken von außen vor dem Euter der Mutter fortbewegen kann, so incidiere man mit einem Beckenmesser die Bauchwandungen des Föten von innen gerade so, daß der Schnitt auf einen der Hinterfüße desselben trifft, erweitere diese Öffnung mit Vorsicht um so viel, daß der Operateur die Hand durchbringen, die Füße einzeln erfassen, heben und wenn nötig nicht anders, in die eigene Bauchhöhle des Fötus führen kann, welchem nächst man nun den Fötus mit Kraft auszieht. »

Gleich wie er gingen Овісн 1865 und Gierer 1865 vor.

\section{B. Das Aus-der-Haut-Ziehen}

Wem die Priorität zukommt, dieses Verfahren erstmals entwickelt zu haben, möchten wir nicht entscheiden. Tatsache ist aber daß es von 1830 an viel geübt wurde.

\section{Instrumente}

Hier spielen vor allem zwei Arten von Instrumenten eine Rolle, das Messer, das offen oder verdeckt sein kann, und der Spatel.

Die Fingermesser gleichen meist dem von Günther 1830, so das von Vix, das der Wiener Schule, das Berner Modell, das Embryotom nach Contamino und viele andere mehr.

Die verdeckten Messer, deren Prinzip darin besteht, daß die Klinge in einer Scheide versteckt werden kann, sollten die Gefahr von Uterusverletzungen beim Einführen des Instrumentes verhindern. Gleich wie bei den offenen Messern ist es uns unmöglich, alle die nur geringgradig voneinander abweichenden Konstruktionen zu erwähnen. Bekanntere verdeckte Messer, die für embryotomische Zwecke Verwendung fanden, waren nach Franck (1876): Das Messer von Günther, der Münchner Sammlung, das von Obermeyer modifizierte Messer nach Hering, das Obermeyersche Geburtsmesser, das Gelenkmesser von Obermeyer, das Embryotom von Brogniez. 
Die Spatel sind ausschließlich für das Aus-der-Haut-Ziehen geschaffene Instrumente. Sie sollten das mühevolle Ablösen der Haut von Hand erleichtern.

So die sogenannte Geburtshohlsonde nach Ungefrohrn (1851). Ein spatelähnliches Instrument mit einer Führungsrille im Stiel für ein speziell dazu konstruiertes Messer.

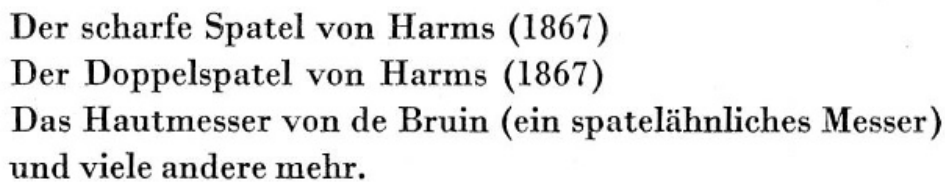

Diese dienten aber außerdem noch zum Abstemmen einzelner Körperteile, wie des Kopfes, des Halses, zum Durchtrennen der Beckensymphyse usw. Hier möge der Meißel nach Margraff genannt werden (1857), der zu dieser Zeit große Zustimmung fand.

Fragen wir uns nun, welche von diesen vielen Instrumenten sich durchsetzen konnten.

Im allgemeinen günstig bewertet wurden das Günthersche verdeckte und vor allem das krumme Fingermesser nach Günther. Baumeister bewertet 1844 das Günthersche verdeckte Messer als «ein sehr brauchbares und empfehlenswerthes Instrument », und Harms 1867 empfiehlt beide. Franck 1876 sagt allgemein über die Fingermesser: «Es gehören diese Messer - die selbstverständlich immer nach der Hand des betreffenden Geburtshelfers gearbeitet sein müssen - zu den einfachsten und zweckmäßigsten Instrumenten für die Embryotomie. «Die verdeckten Messer» stehen jedoch ... im Gebrauche den Fingermessern bedeutend nach, obgleich man auf den ersten Blick sie für überaus zweckmäßig hält. Es sind namentlich zwei Umstände, die ihren Gebrauch erschweren, nämlich die Unmöglichkeit, im Uterus die Finger der Hand in gewöhnlicher Weise zu gebrauchen und den Einfluß der Fruchtwässer und Fruchthüllen auf den Mechanismus dieser Instrumente.»

Wieviele Instrumente der einzelne Geburtshelfer gebrauchte, hing ganz von dessen praktischen Fähigkeiten und seiner theoretischen Interessiertheit ab. Ganz allgemein läßt sich jedoch feststellen, daß mit fortschreitender Zeit die Auswahl der empfohlenen Instrumente immer kleiner wurde.

Binz, 1830, der nur die Amputation kannte, empfahl:

die große Zahnzange,

die kleine Zahnzange, 


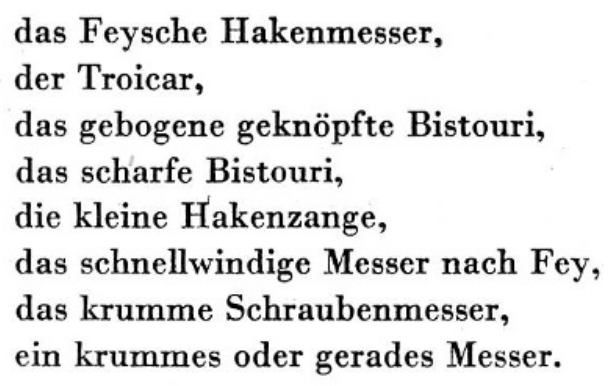

BAUMEISTer erwähnt 1844:

$$
\begin{aligned}
& \text { die große und kleine Zahnzange von Binz, } \\
& \text { die Feysche schnellwindige Schraubenzange, } \\
& \text { das Feysche Hakenmesser, } \\
& \text { das lange Scheerenmesser nach Günther, } \\
& \text { das krumme Fingermesser, } \\
& \text { das verdeckte Messer nach Günther. }
\end{aligned}
$$

Nur über das letzte äußert er sich als «ein sehr brauchbares und empfehlenswertes Instrument», während er bei den übrigen stets den Gebrauch einschränkende Bemerkungen macht.

FrancK 1876 macht die schon oben angegebenen günstigen Bemerkungen über das verdeckte und offene Messer und empfiehlt den Margraffschen Meißel (oder auch Stemmeisen genannt) als außerordentlich zweckmäßig. Dagegen können nach ihm sehr wohl entbehrt werden das krumme Hakenmesser, der Geburtshaken mit verborgenem Messer und «eine Reihe älterer Instrumente, wie das große Günthersche Scherenmesser, das Feysche Hakenmesser, die große Binzsche Zahnzange, die schnellwindige Feysche Schraubenzange», «da sie zum Theil sehr umfangreiche, komplizierte und kostspielige Instrumente» sind und «meist nur in ganz bestimmten Fällen Anwendung finden und sind dann leicht durch einfachere Mittel zu ersetzen.»

Deneubourg, 1880, ein belgischer praktischer Tierarzt, aber kommt mit einem Minimum an Instrumenten für alle Geburten aus. «Les instruments que nous avons mis en usage pendant notre longue pratique sont: $1^{\text {er }}$ un petit lac... $2^{\mathrm{e}}$ un petit crocher de boucher, $3^{\mathrm{e}}$ un bistouri convexe et $4^{\mathrm{e}}$ un bistouri à serpette.»

\section{Indikationen}

Die Embryotomie ist stets noch eine gefährliche Operation geblieben, hat aber doch schon viel von ihrer Schwere eingebüßt. Deshalb wurde sie auch häufiger. 
«Es ist nichts bezeichnender für das dermalige Entwicklungsstadium der thierärztlichen Geburtshülfe, als die Häufigkeit, mit welcher die Embryotomie hier heut zu Tage angewandt wird. Man bezeichnet sogar die Ausbildung der Embryotomie als einen entschiedenen Fortschritt der thierärztlichen Geburtshülfe ... Es unterliegt wohl keinem Zweifel, daß auch in der Veterinärgeburtshülfe bessere Entwicklungsmethoden die Embryotomie mehr und mehr verdrängen werden ... Die Embryotomie ist demnach überall da angezeigt, wo entweder in Folge von fehlerhaften Lagen oder abnormer Beschaffenheit des Jungen eine Lageberichtigung und Extraktion im ganzen nicht möglich, oder wo bei bestehendem Tod des Jungen als das für das Mutterthier weniger eingreifende Verfahren erscheint ...» «Die Embryotomie bleibt immer eine für das Mutterthier sehr bedeutungsvolle, ja gefährliche Operation » (Franck 1876).

Gleich äußert sich SAINT-Cyr (1875): «Le débutant est tenté de se dire qu'après tout, l'embryotomie ne parait pas être une opération bien difficile; le practicien, au contraire, est tenté de se demander, si ceux qui la décrivent ainsi ont jamais mis la main dans l'uterus d'une jument ou d'une vache en travail de parturition. Il sait, en effet, par expérience, que la pluspart des manœuvres si complaisamment et si exactement décrites, sont absolument inéxecutables.»

\section{Die einzelnen subcutanen Methoden}

Wie oben schon erwähnt, möchten wir die Prioritätsfrage nicht entscheiden. Wir können uns aber einer ganz allgemeinen Bemerkung nicht enthalten.

Das Ungenügen und die Gefährlichkeit der Amputation drängten nach einer anderen Lösung. Die entschiedenen Fortschritte, die die Tiergeburtshilfe seit Jörg gemacht hatte, und der Umstand, daß die Geburtshilfe immer mehr Teilgebiet der tierärztlichen Praxis, also von Leuten mit naturwissenschaftlicher Ausbildung, wurde, ermöglichten ein erfolgreiches Suchen (siehe Jörg, der sich beklagt, daß die Geburtshilfe hauptsächlich von Hirten und anderen Quacksalbern vorgenommen werde und in der tierärztlichen Kunst noch lange nicht die ihr gebührende Beachtung gefunden habe). Es darf deshalb nicht verwundern, daß gleichzeitig und an verschiedenen Orten gleiche Versuche durchgeführt wurden.

Entscheidend für die Einschätzung der Bedeutung einer Methode bleibt immer der Widerhall, den sie bei den Fachleuten findet. Deshalb setzen wir als Wendepunkt der Methode das Jahr 1830 an. 
1830 ist aber nicht das Geburtsjahr der neuen Methode, denn Versuche dazu wurden schon früher unternommen, blieben aber in der Fachwelt unbeachtet und wurden wieder vergessen.

So soll nach Thomassen (c. Saint-Cyr und Violet 1888) schon 1804 Le France van Berkhey über eine ähnliche Operation an den Vorderbeinen berichtet haben, die einerseits von J. BLANkEN mit einem « Bistouri caché à ressort» und einem Spatel und andererseits von einem J. LAUwen mit einem "petit couteau » oder «bistouri à lame concave et à manche court » ausgeführt worden sei (siehe auch 1. Etappe, S. 41, Knip Vater und Sohn).

Tatsache ist aber, daß das Aus-der-Haut-Ziehen erst von 1830 an durch HuvelLIER und unabhängig davon durch GüNTHER in die Geburtshilfe eingeführt wurde. Von da an haben sich dann eine große Anzahl von Tierärzten darüber geäußert und dieses Vorgehen als Methode der Wahl bezeichnet.

Günther beschreibt seine Methode folgendermaßen : «Liegt aber das Junge zu fest und kann die Operation auf vorstehende Weise» (Amputation) "wegen Mangel an Raum nicht beschafft werden, so ziehe man einen Schenkel scharf hervor, löse die Haut von der Röhre an aufwärts so hoch, als irgend wie möglich, durchschneide auch am Rumpfe, womöglich, die Brustmuskeln, schlaufe dann die Schenkel an, und lasse ihn durch ein paar tüchtige Gehülfen im eigentlichen Sinne aus der Haut ziehen.»

Huvelliers Verfahren ist im wesentlichen dasselbe. Die nun noch vorgenommenen Abweichungen betrafen nur mehr Kleinigkeiten. So zogen die einen Geburtshelfer vor, zuerst den Längsschnitt und dann erst den Zirkelschnitt zu machen, die andern aber führten den Zirkelschnitt vor dem Längsschnitt aus. Wieder andere führten den Längsschnitt von zentral nach peripher, statt von peripher nach zentral, oder machten den Zirkelschnitt entweder schon am Fessel oder an der Röhre oder noch weiter oben, oder machten zuerst statt einen ${ }^{4} / 4$ - nur einen ${ }^{3} / 4$-Zirkelschnitt usw.

Im folgenden geben wir nun noch, chronologisch geordnet, das Verfahren einzelner Geburtshelfer an:

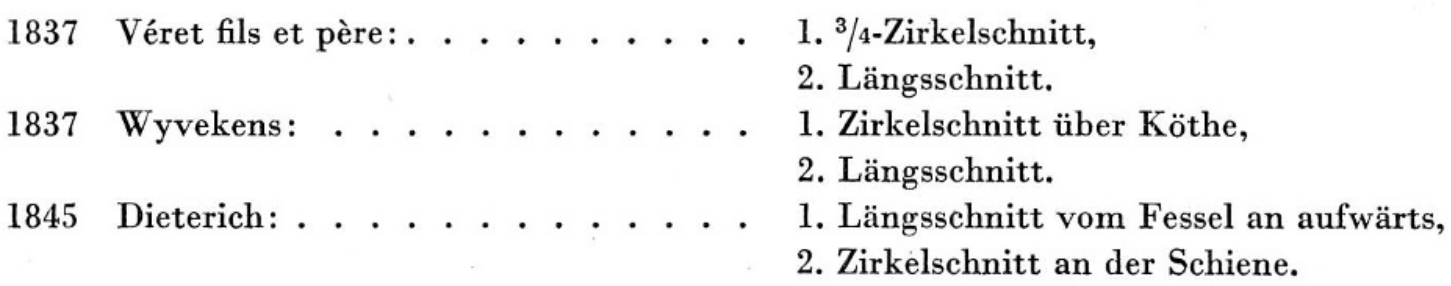


Hierholzer: . . . . . . . 1. Längsschnitt von der Schulter an abwärts zur Klaue,

2. Zirkelschnitt.

1848 John Nelson: . . . . . . . . . . 1. Längsschnitt bis zur Schulter,

2. Zirkelschnitt am Knie.

? Engesser und Schüttemüller: . . . . . Zirkelschnitt möglichst hoch.

1851 Ungefrohrn: . . . . . . . . . . 1. Zirkelschnitt Mitte Röhre,

2. Längsschnitt.

1853 Baumeister: . . . . . . . . 1. Längsschnitt nach abwärts,

2. Zirkelschnitt.

In der ersten Auflage wird expressis verbis die subcutane Methode nirgends erwähnt.

1867 Harms: . . . . . . . . . 1. ${ }^{3 / 4}$-Zirkelschnitt,

2. Längsschnitt,

3. 1/4-Zirkelschnitt.

1875 Saint-Cyr: . . . . . . . . . 1. Zirkelschnitt über Carpus,

2. Längsschnitt medial oder lateral.

1876 Franck: . . . . . . . . . . . 1. Längsschnitt,

2. Zirkelschnitt über Köthe.

Bei Franck findet sich unter anderem erstmals die Bemerkung, man solle den noch lebenden Föten vor dem Eingriff töten.

\section{Etappe}

Wie wir schon in der Einleitung bemerkt haben, kann diese Etappe nur sehr summarisch behandelt werden.

Sie ist hauptsächlich durch die Anwendung sägender Instrumente gekennzeichnet. Mit Hilfe von Sägen wurde die früher sehr mühevolle, zweieinhalb Stunden und länger dauernde Zerstückelung wesentlich erleichtert und abgekürzt, und die Gefahren wurden kleiner. Dies erklärt wohl die Operationsfreudigkeit, die im Beginn manche Geburtshelfer befiel. So gibt Tierarzt Wollesen (1902) an, von 1893 bis 1901 im ganzen bei 346 Kühen und Stuten Geburtshilfe geleistet zu haben. Davon hatte er 232 durch Embryotomie und nur 114 ohne Embryotomie entbunden! Was hätte Jörg dazu gesagt?

An Sägen lassen sich drei Gruppen unterscheiden:
1. Sägen mit Handgriff,
2. Kettensägen,
3. Drahtsägen.

Daneben sind in dieser Etappe noch Instrumente zu nennen, die nach Art eines Ecraseurs arbeiteten, solche, die zum Heranziehen von Körperteilen 
dienten (Doppelbohrer nach Kaiser 1894) und der Rachiofor und Pelviklast nach Stüwen 1915.

1875 wurde die Fachwelt durch Persson mit der Erfindung eines schwedischen Tierarztes, SJöstedT, bekanntgemacht, der Kettensäge. Wer jedoch glaubt, diese Säge hätte gleich einen gewaltigen Umschwung in der Embryotomie bewirkt, irrt sich. In den nordischen Ländern freilich kam sie bald zu Ansehen. Im deutschen und französischen Sprachgebiet aber stund man ihr mit Skepsis gegenüber. Die meisten Lehrbücher von 1880 bis 1900 erwähnen die Kettensägen nur beiläufig und ohne eigene Erfahrung (so z. B. Frank 1876, Saint-Cyr et Violet 1888, Deneubourg 1880 nennt sie überhaupt nicht, Harms 1897, de Bruin). Dafür wurden verschiedene andere Instrumente geschaffen, wie das nach dem Prinzip eines Ecraseurs arbeitende Pflanzsche Embryotom 1896 (Verbesserung von Pflanz selber 1897), über das sich 1898 Schmidt-Buxtehude sehr lobend äußert. Oder man bediente sich der Sägen mit Handgriff: Säge von Hinze, die nach Wollesen die erste geburtshilfliche Säge gewesen sein soll, Säge von Oehmke 1892, Säge von Koiranski 1892. Albrecht 1896 gebrauchte eine gewöhnliche Lochsäge, die er sich «vom Wagner des Ortes» anfertigen ließ.

Die Wende trat erst um die Jahrhundertwende ein. Von nun an häuften sich günstige Meldungen auch in deutschen Zeitschriften (Marsch-Wilstet 1894, Wollesen 1902, Schiel 1904 und andere).

BeHм hatte dadurch daß er an die einzelnen Kettenglieder vorstehende Zacken anbrachte, den Mangel des schlechten Angreifens zu beheben versucht, und Sснӧтteler konstruierte ein Führungsrohr dazu.

Die heute meistgebrauchten Sägen, die Drahtsägen, gehen auf voN StaA zurück. Ihnen haftete zu Beginn der Nachteil des schnellen Durchreißens an. Diesen Fehler suchte 1903 Ehlers dadurch zu umgehen, daß er statt Kupfer- Stahldraht nahm.

Aber schon Franck gibt 1876 Versuche mit Drahtsägen bekannt. Es ist dies, soviel uns bekannt ist, die erste schriftliche Mitteilung über Drahtsägen. «Zum Schlusse möchte ich noch einige Versuche nicht unerwähnt lassen, die ich zu dem Zwecke gemacht habe, um zu sehen, ob man nicht durch Draht den Kopf oder Rumpf eines Kalbes oder Fohlens durchschneiden kann. Es hat sich dabei herausgestellt, daß dies mit dünnem, gut geglühtem Eisen- oder Messingdraht tatsächlich möglich ist. Die Weichgebilde, selbst das Nackenband werden ziemlich rasch durchschnitten, schließlich selbst der Knochen. In einigen Fällen wurde ein Wirbel in der Mitte durchschnitten, in einem anderen Falle war der Zahnfortsatz des zweiten Hals- 
wirbels, in einem dritten waren beide Condylen des Oberhauptbeines abgeschnitten. Ja, der ganze Brustkasten konnte durchschnitten werden, und zwar mit Haut, Muskeln und Knochen. Es wurden hierzu dünne, einfache oder zu zwei oder drei zusammengedrehte feinere und möglichst lange Drähte (3-4 Meter lang) verwendet, und durch zwei Mann in sägenden Bewegungen gegen den Rumpf, Hals und Kopf gezogen. Neusilberdraht erwies sich haltbarer als Messingdraht. Soviel versprechend diese Versuche auch waren, so dürfte für die Praxis vorerst doch nur ein geringer Nutzen abfallen, und zwar deshalb, weil die Drähte immer 2-10 mal entzweirissen, ehe die Amputation des betreffenden Gliedes vollendet war. In Wirklichkeit dürfte es aber schwer halten, immer einen neuen Draht in die bereits begonnene Wunde einzuführen. Es ist übrigens recht wohl möglich, daß ein besserer Draht diesem Übelstande abhilft. Der Hauptgrund, warum der Draht so leicht riß, lag jedenfalls darin, daß er sich außerordentlich erhitzte. Nach einigen Sägebewegungen schon stieg Dampf in die Höhe, und der Draht wurde brennend heiß. Je kürzer der Draht, um so rascher erhitzte er sich und um so rascher riß er. Bei der Anwendung in praxi müßte man jedes Drahtende durch eine Blechröhre ziehen, um Verletzungen des Mutterthieres zu verhindern ... Obgleich die Resultate mit Draht, wie angegeben, nicht derart sind, daß sie dieser Methode eine allgemeine Verwendung für die Embryotomie sichern könnten, so zeigen sie doch, daß durch Draht ein ganzes Junges zerschnitten werden kann. Ich halte schon diese Thatsache für wichtig genug, die Versuche weiter fortzusetzen. Die Drahtsäge wäre ja ein so einfaches und wohlfeiles, überdies überall anzuwendendes Mittel, daß damit eine ganze Reihe Instrumente erspart werden könnten.»

Führungsrohre für Drahtsägen haben unter anderen konstruiert:

1906 Neubart (Einröhrenapparat), 1921 Thygessen (Zweiröhrenapparat).

Damit möchten wir unseren Beitrag zur Embryotomiegeschichte schlieBen.

\section{Schriftum}

Absyrthus, Siehe Baranski, 1886.

M. Albrecht, Geburtshülfliches, Wochenschrift für Tierheilkunde und Viehzucht, von Th. Adam, 33 (1886) 260.

- Geburtshilfe beim Pferd, Handbuch der tierärztlichen Chirurgie und Geburtshilfe, Wien und Leipzig 1913. 
Herm. ANacker, Embryotom und Geburtshacken nach Contamino, Der Thierarzt, Monatsschrift von Anacker 12 (1873) 211.

M. Aubry, De quelques cas de dystocies, Recueil de médecine vétérinaire XXXVI (1859) 721. Anton Baranski, Geschichte der Thierzucht und Thiermedizin im Altertum, Wien 1886.

W. Baumeister, Die thierärztliche Geburtshülfe. Ein Handbuch, Stuttgart 1844.

W. Baumeister und A. RuefF, Die thierärztliche Geburtshülfe. Ein Handbuch, 3. Auflage, Stuttgart 1853.

- Die thierärztliche Geburtshülfe. Ein Handbuch, 5. Auflage, Stuttgart 1869.

W. BECKER, Ein embryotomisches Verfahren, Berliner tierärztliche Wochenschrift 1892, 282.

Pantaleon Binz, Theoretisch-praktische Geburtshülfe für die Haussäugethiere, Freiburg 1830.

M. G. De Bruin, Die Geburtshilfe beim Rind, 3. Auflage, Wien und Leipzig 1910.

HeINrich Buess, Geschichtlicher Überblick über die Entwicklung der Geburtshilfe, in TH. KolLER, Lehrbuch der Geburtshilfe und Gynäkologie, Basel 1948.

Colerus, siehe Dieterichs, 1845.

M. CANU, Recueil de médecine vétérinaire 1837.

M. CANU, Quelques réflexions sur l'obstétrique, Recueil de médecine vétérinaire XXXVII (1860) 161.

Columella, siehe Baranski, 1886.

O. Delafond, Rapport à la Société centrale de médecine vétérinaire, Recueil de médecine vétérinaire XXVI (1849) 359.

Fr. Deneubourg, Traité pratique d'obstétrique, Bruxelles 1880.

Louis Devraigne, Histoire de l'obstétrique, in Histoire générale de la Médecine, par Laignel-LaVastine, tome 3, Paris 1949.

J.F.C.Dieterichs, Handbuch der praktischen Geburtshülfe bei den größeren Haussäugethieren, Berlin 1845.

Paul Diepgen, Geschichte der Medizin, Band 1, Berlin 1949.

DonnarieIx, Du part contre nature dans la jument, Recueil de médecine vétérinaire XXVI (184.9) 207.

- Nouvelles observations sur quelques cas de dystocies, Recueil de médecine vétérinaire XXXVII (1860) 577.

- Part contre nature dans la jument, Recueil de médecine vétérinaire XXXIV (1857) 661.

- Recueil de médecine vétérinaire XXXV (1858) 190.

- Sur quelques cas de part contre nature chez le jument ... Recueil de médecine vétérinaire XXXVI (1859) 321.

J.B.N.C.Drouard, Accouchement contre nature, Recueil de médecine vétérinaire XIX (1842) 40, 131.

Sebastian Fey, Die künstliche Zerstückelung und Ausziehung schwerster regelwidriger Geburten bei landwirthschaftichen größeren Haussäugethieren, Constanz 1823.

L. Franck, Beiträge zur Geburtshülfe bei unseren Hausthieren, Wochenschrift für Tierheilkunde und Viehzucht, von Tн. АDAm, 18 1874, 2, 245.

- Handbuch der thierärztlichen Geburtshiilfe, Berlin 1876.

L. Franck, Рн. Göring, Handbuch der thierärztlichen Geburtshilfe, 2. Auflage, 1887.

L. Franck, M. Albrecht und Th. Oppermann, Handbuch der tierärztlichen Geburtshilfe, 6. Auflage, Berlin 1922. 
Joh. Dav. Gierer, Magazin von Gurlt und Hertwig, 1865.

- Österreichische Vierteljahrsschrift für wissenschaftliche Veterinärkunde 1867.

- Österreichische Vierteljahrsschrift für wissenschaftliche Veterinärkunde 1871.

J.H.Fr. GüNThER, Thierärztliche Geburtshülfe, 1830.

Carsten HaRMs, Lehrbuch der thierärztlichen Geburtshülfe, Hannover 1867.

L.E.HüBNER, Einige Abhandlungen über schwere Geburten, Zeitschrift für die gesamte Tierheilkunde und Viehzucht, von Dieterichs, Vix und Nebel, 1847, 14, 77.

Michael Harward, c. Rieck, 1932.

Hierholzer, Veterinärbericht des Thierarztes Hierholzer, Referat in Zentralblatt für die gesamte Veterinärmedizin, von Kreutzer, II (1846) 108.

A. Hirsch, Biographisches Lexikon berühmter Ärzte aller Zeiten ..., 2. Auflage, Wien 1929-1935.

Hurtrel D'Arboval, Wörterbuch der Thierheilkunde. Nebst Anmerkungen von Dr. Th. RENNER, Weimar 1831.

J. Chr. Gottrr. Jörg, Anleitung zu einer rationnellen Geburtshülfe der landwirthschaftlichen Thiere für Thierärzte, gebildete Ökonomen und Geburtshelfer, 2. Auflage, Leipzig 1818.

- Vierzehn Kupfertafeln nebst Erklärung zur Geburtshülfe der landwirthschaftlichen Thiere, Leipzig 1818.

HeINR. KAISER, Doppelbohrer, ein Instrument zur Geburtshilfe, Berliner tierärztliche Wochenschrift 1894, 378.

Joh. Adam Kersting, siehe de Bruin, 1910.

M. KoIrANSKy, Ein neues Instrumententaschenbesteck zur Ausführung der Embryotomie, Monatshefte der praktischen Tierheilkunde XIII (1902) 501.

Fr. Lausch, Magazin, von Gurlt und Hertwig, 8 (1842) 463.

MargrafF, Wochenschrift für Tierheilkunde und Viehzucht, von Th. Adam, 1 (1857) 297.

MARTy, Referat in Journal des Vétérinaires du Midi XIV (1858) 400.

Mignon, Rapport à la Société centrale de médecine vétérinaire, Recueil de médecine vétérinaire XXVI (1849) 188.

Franz Carl Müller, Geschichte der organischen Naturwissenschaften im 19. Jahrhundert, Berlin 1902.

John Nelson, siehe Palat, 184.9.

L. G. Neumann, Biographies vétérinaires, Paris 1896.

M. Овісн, Geburtshülfliches, Wochenschrift für Tierheilkunde und Viehzucht, von Tн. Adaм, $12(1868) 345$.

Oeнмкe, Die Embryotomie und neuere Hilfsmittel zur Ausführung derselben, Berliner tierärztliche Wochenschrift 1892, 217.

PaLAt, Parturition contre nature chez une vache, Recueil de médecine vétérinaire XL (1863) 28.

PässLer, Jahresbericht über das Veterinärwesen Sachsen 1861, 122.

Pflanz, Embryotom zur vollständigen Durchschneidung des Jungen im Mutterleibe, Berliner tierärztliche Wochenschrift 1896, 556.

- Verbessertes Embryotom, Berliner tierärztliche Wochenschrift 1897, 621.

J. RAINARD, Traité complet de la parturition des principales femelles domestiques, Lyon 1850.

Recordon, Présentation transversale d'un foetus-Embryotomie, Recueil de médecine vêtérinaire 1877. 
RENNER, siehe Hurtrel d'Arboval, 1831.

Richter-Goetze, Lehrbuch der Tiergeburtshilfe, Berlin 1950.

WilH. Rieck, Das Veterinärinstrumentarium im Wandel der Zeiten, Jubiläumskatalog Hauptner, Berlin 1932.

M. E. RocharD, De quelques erreurs commises dans un travail sur l'obstétrique vétérinaire, Recueil de médecine vétérinaire XXXVII (1860) 18.

F. SAINT-Cyr, Traité d'obstétrique vétérinaire, Paris 1875.

F. Saint-Cyr et Тн. Violet, Traité d'obstétrique vétérinaire, $2^{\mathrm{e}}$ édition, Paris 1888.

M. C.SAMson, Opération (vaginotomie) souvent indispensable après la part labourieux, Recueil de médecine vétérinaire XL (1863) 106.

G. W.Schrader, Biographisch-literarisches Lexikon der Thierärzte aller Zeiten ..., Stuttgart 1863.

Schiel, Die Embryotomie mit der Perssonschen Kettensäge, Deutsche tierärztliche Wochenschrift 12 (1904) 221.

Јон. Јак. Schlumpf, Aus meiner Praxis, Schweizer Archiv für Tierheilkunde I (1816) 55.

Sснмidt-Buxtenude, Das Pflanzsche Embryotom, Deutsche tierärztliche Wochenschrift 6 (1898) 241.

UNGEFROHRN, Anwendung und Beschreibung eines Instrumentes, einer neu erfundenen Geburtshohlsonde, Zeitschrift für die gesamte Tierheilkunde und Viehzucht, von VIX und NEBEL, 17 (1851) 240.

Hermann Vitet's Unterricht in der Vieharzeneykunst, übersetzt von Jон. Christian Polycarp Erxleben und Wilh. Joh. Conrad Hennemann, 3 Bände, Lemgo 1773, 1785, 1786.

J. WALthER, Jahresbericht über das Veterinärwesen Sachsen 1861, 122.

Max Wegscheider, Geschichte der Geburtshilfe, im Handbuch der Geschichte der Medizin, von Neubauer, Band 3, Jena 1903.

WILHELM, Jahresbericht über das Veterinärwesen Sachsen 41 (1897) 14.7.

Chr. Wollesen, Ein Beitrag zur Geschichte und Technik der Embryotomie, Monatshefte für praktische Tierheilkunde 13 (1902) 481. 